\title{
N
}

NASA TECHNICAL MEMORANDUM

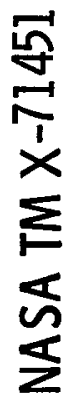

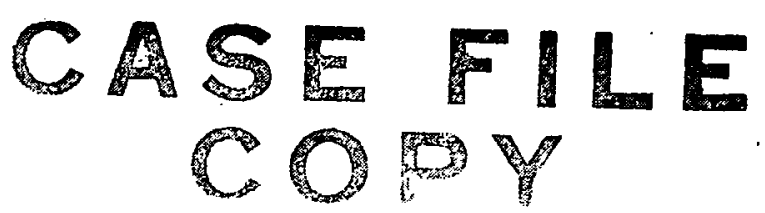

\section{DISPERSION AND DILUTION OF JET AIRCRAFT EXHAUST AT HIGH-ALTITUDE FLIGHT CONDITIONS}

by James D. Holdeman

Lewis Research Center

Cleveland, Ohio 44135

TECHNICAL PAPER proposed for presentation at

Twelfth Aerospace Sciences Meeting sponsored

by the American Institute of Aeronautics and Astronautics

Washington, D. C. , January 30-February 1, 1974 


\author{
James D. Holdeman \\ National Aeronautics and Space Administration \\ Lewis Research Center \\ Cleveland, Ohio
}

\section{Abstract}

A method is presented for estimating the dispersion and dilution of jet aircraft exhaust from aircraft passage through times on the order of weeks thereafter. In the near wake of the aircraft, the solution is that for round turbulent jets in a parallel flow. More rapid dispersion due to atmospheric effects begins when the scale-dependent eddy viscosity becomes larger than the turbulent jet eddy viscosity. In the far wake region, the solution approaches that for scale-dependent dispersion from a point source moving with the afrcraft. Calculations are presented for supersonic aircraft at high-altitude flight conditions.

\section{Introduction}

Concern about the possible adverse impact of a fleet of high altitude aircraft on the environment has motivated interest in aircraft wakes and stratospheric chemical kinetics. The significance of chemical reactions in aircraft wakes is expected to be dependent on atmospheric conditions and on the concentrations of possible reactants. The latter, of course, is determined by the engine exhaust emissions and their dispersion into the atmosphere.

Aspects of the dispersion in regions dominated by the aircraft initial conditions have been considered in References 1 and 2 . The dispersion in regions dominated by ambient atmospheric conditions has been discussed in Reference 3. The method presented in this report extends the analysis of Reference 2 to include atmospheric dispersion effects and mult1ple engine aircraft conflgurations. At long times after aircraft passage, the solution approaches the result for scale-dependent dispersion from a point source moving with the aircraft.

Although the solution approached in the far wake region using the present method is similar to that given in Reference 3 , the present method does not require knowing the time after aircraft passage at which atmospheric effects become important or the plume size and concentrations at that time. Since calculations are begun at the engine exhaust plane, the initial conditions required for the method of Reference 3 are included in the results obtained using the present method.

\section{Analysis}

The dispersion of the exhaust of a turbojet engine at flight conditions is modeled as a round jet mixing with a parallel flow. The Prandt 1 and Schmidt numbers throughout the flow are assumed to be equal to 1 , thus the conservation equations for momentum, total enthalpy, and species concentration are identical. This permits the problem to be solved by considering only the continuity and momentum equations.

The integral momentum equation, evaluated across the entire width of the flow, is

$$
\int_{0}^{\infty} \rho u_{x}\left(u_{x}-u_{\infty}\right) r d r=\rho_{j} u_{j}\left(u_{j}-u_{\infty}\right) \frac{r_{j}^{2}}{2}
$$

where $u_{x}$ is the local streanwise velocity, $u_{\infty}$ is the flight speed, $u_{j}$ is the jet exhaust velocity, $r_{j}$ is the radius of the jet at the engine exhaust plane, and $\rho$ is the gas density. All symbols are defined in the appendix.

A second relation is obtained by evaluating the integral momentum relation at the half-value radius, $r_{5}$,

$$
\begin{array}{r}
\frac{d}{d x} \int_{0}^{r_{5}} \rho u_{x}^{2} r d r=\left(\frac{u_{c}+u_{\infty}}{2}\right) \frac{d}{d x} \int_{0}^{r_{5}} \rho u_{x} r d r \\
+r_{5}\left[\left.\varepsilon \frac{\partial u_{x}}{\partial r}\right|_{r=r_{5}}\right]
\end{array}
$$

where $r_{5}$ is the radius at which the local velocity is one-half the sum of the centerline velocity, $u_{c}$, and the flight speed, $u_{\infty}$. A gradient diffusion model for the shear stress is assumed. The eddy viscosity, $\varepsilon$, in the near wake region is assumed to be directly proportional to the jet mass flow plus the absolute value of the entrainment mass flow and inversely proportional to the jet width. The resultant eddy viscosity relation, from Reference 2, is

$$
\varepsilon=\frac{0.036\left\{\rho_{j} u_{j} \frac{r_{j}^{2}}{2}+\left|\int_{0}^{\infty}\left(\rho u_{x}-\rho_{\infty} u_{\infty}\right) x d r-\left(\rho_{j} u_{j}-\rho_{\infty} u_{\infty}\right) \frac{r_{j}^{2}}{2}\right|\right\}}{\left.r_{g}(1.0+0.6] M_{c}-M_{\infty} \mid\right)}
$$

The equations are closed by assuming that the velocity difference and concentration difference ratios are Gaussian, that is,

$$
\frac{c-c_{\infty}}{c_{j}-c_{\infty}}=\frac{u_{x}-u_{\infty}}{u_{j}-u_{\infty}}=\left(\frac{u_{c}-u_{\infty}}{u_{j}-u_{\infty}}\right) \exp \left[-\lambda\left(\frac{r}{r_{5}}\right)^{2}\right]
$$

where $\lambda=\ln 2$. Equations (1) to (4) plus the perfect gas law provide a complete set of equations for the solution of the jet spreading and dilution. The integrals in Equations (1) to (3) may be evaluated in closed form at any streamwise location. The jet width is obtained in terms of the centerline velocity with Equation (1). The incremental changes in streamwise distance corresponding to selected incremental changes in the centerline velocity are obtained with Equation (2). The streamwise distance, $x$, corresponding to any desired $u_{c}$ 
must then be obtained by numerical integration. If desired, the spreading and dilution results way be expressed in terms of the time after alrcraft passage rather than the downstream distance. In this case, $t=x / u_{\infty}$, where $u_{\infty}$ is the flight speed.

At downstream distances where $\rho \cong \rho_{\infty}$ and $\left(u_{x}-u_{\infty}\right) \ll u_{\infty}$, Equations (1) and (4) provide the following relation between the half-value width and the centerline concentration difference ratio,

$$
\frac{c_{c}-c_{\infty}}{c_{j}-c_{\infty}}=\lambda \frac{\left(\frac{\rho_{j} u_{j}}{\rho_{\infty} u_{\infty}}\right) r_{j}^{2}}{r_{5}^{2}}
$$

where $c_{c}$ is the concentration on the centerline at any downstream location. In this regton the asymptotic result for a small excess jet is obtained, namely

$$
\begin{gathered}
\frac{c_{c}-c_{\infty}}{c_{j}-c_{\infty}} \sim x^{-2 / 3} \\
r_{5}-x^{1 / 3}
\end{gathered}
$$

and the eddy viscosity is decreasing with downstream distance, 1.e.,

$$
\epsilon \sim \mathrm{x}^{-1 / 3}
$$

Results for the dispersion of both large and small turbojet engines from the engine tailpipe through the above region are given in Reference 2 .

Multiple engine alrcraft are treated by superimposing the concentration distributions from the several engines. In the case of a twin-jet aircraft the concentration difference ratio distribution is given by

$$
\begin{aligned}
\frac{c-c_{\infty}}{c_{j}-c_{\infty}}= & \left(\frac{c_{c}-c_{\infty}}{c_{j}-c_{\infty}}\right) \exp \left[-\lambda\left(\frac{z}{r_{5}}\right)^{2}\right] \\
& \times\left\{\exp \left[-\lambda\left(\frac{y-\frac{E C L}{2}}{r_{5}}\right)^{2}\right]+\exp \left[-\lambda\left(\frac{y+\frac{E C L}{2}}{r_{5}}\right)^{2}\right]\right\}
\end{aligned}
$$

where $y$ and $z$ are respectively the horizontal and vertical distances from the aircraft centerline, and ECL is the horizontal separation between engines. When the spreading of the exhaust from each engine is small with respect to the distance between engines, there is no significant interaction. When the half-value radius approaches the engine separation distance, the concentration downstream from each engine is increased from what it would be alone due to the contribution from the other engines. As $r_{5}$ becomes much larger than the engine separation, the concentration downstream from each engine is nearly equal to the number of engines times the concentration from each.

The dispersion described above is controlled by aircraft and engine effects. The eddy viscosity, $E$, in this region is decreasing with increasing distance from the aircraft (see Eq. (8)), and at some distance 1 ts value becomes less than the eddy viscosity for scale-dependent diffusion in the atmosphere. When this occurs, the atmospheric effects are assumed to be dominant and the scaledependent eddy viscosity is used in all subsequent calculations. Up to this point, the exhaust of each engine is assumed to decay axisymetrically. However, when atmospheric effects are dominant, the plume dispersion in the horizontal plane is known to be greater than the vertical dispersion. $(3,4)$ Accordingly, a blvariant Gaussian distribution is assumed for the dispersion of the exhaust from each engine. That is,

$\frac{c-c_{\infty}}{c_{j}-c_{\infty}}=\left(\frac{c_{c}-c_{\infty}}{c_{j}-c_{\infty}}\right) \exp \left\{-\lambda\left[\left(\frac{y}{y_{5}}\right)^{2}+\left(\frac{z}{z_{5}}\right)^{2}\right]\right\}$

where $y_{5}$ and $z_{5}$ are the horizontal and vertical half-value widths, respectively. For a twin-jet aircraft, the concentration distribution in the plume is given by

$$
\begin{aligned}
& \frac{c-c_{\infty}}{c_{j}-c_{\infty}}=\left(\frac{c_{c}-c_{\infty}}{c_{j}-c_{\infty}}\right) \exp \left[-\lambda\left(\frac{z}{z_{5}}\right)^{2}\right] \\
& \quad\left\{\exp \left[-\lambda\left(\frac{y-\frac{E C L}{2}}{y_{5}}\right)^{2}\right]+\exp \left[-\lambda\left(\frac{y+\frac{E C L}{2}}{y_{5}}\right)^{2}\right]\right\}
\end{aligned}
$$

where $\left(c_{c}-c_{\infty}\right) /\left(c_{j}-c_{\infty}\right)$ is the concentration difference ratio on the engine centerline for each engine. Similar expressions may be formulated for other multiple engine aircraft configurations.

The solution method used in the region where atmospheric effects are dominant is the same as the method used in the near wake except that equation (10) is used in place of equation (4), and the atmospheric scale-dependent eddy viscosity is used in place of the turbulent jet eddy viscosity. The atmospheric eddy viscosity is formulated using the diffusion coefficients given in Reference 3, namely

$$
K=\frac{1}{\frac{1}{\left(D^{1 / 3} \sigma^{4 / 3}\right)}+\frac{1}{K_{m}}}
$$

where

$K_{m}=K_{z}=1 \frac{m^{2}}{\sec }$ for vertical dispersion

$K_{m}=K_{y}=5 \times 10^{5} \frac{m^{2}}{\mathrm{sec}}$ for hortzontal dispersion

$\mathrm{D}=2 \times 10^{-4} \mathrm{~m}^{2} / \mathrm{sec}^{3} ;$ atmospheric dispersion rate

$\sigma=z_{5} / \sqrt{2 \lambda}$ for vertical diffusion

$\sigma=y_{5} / \sqrt{2 \lambda}$ for horizontal diffusion

The diffusion coefficients given by Equation (12) represent the kinematic eddy viscosity. Thus $\varepsilon=\rho K$.

The plume width varies continuously from the jet dominant region into the atmospheric dominant region. In this latter region the plume width approaches the solution obtained by assuming scale 
dependent dispersion from a point source moving with the alrcraft. That 18 .

$$
\frac{z_{5}^{2}}{2 \lambda K_{z}}+\frac{3 z_{5}^{2 / 3}}{(2 \lambda D)^{1 / 3}}=2 t
$$

for vertical dispersion, and

$$
\frac{y_{5}^{2}}{2 \lambda K_{y}}+\frac{3 y_{5}^{2 / 3}}{(2 \lambda D)^{1 / 3}}=2 t
$$

for horizontal dispersion. This solution is stmilar to that given in Reference 3. In this region the horizontal and vertical plume widths are independent of the aircraft configuration, flight speed, the engine power setting (afterburning or nonafterburning), and the number of engines.

Since in the far-wake the velocity difference is small compared to the flight speed, and the density of gases in the plume 18 nearly equal to the amblent density, equations (1) and (10) provide the following relation between the centerline concentration difference ratio and the vertical and horizontal half-value widths.

$$
\frac{c_{c}-c_{\infty}}{c_{j}-c_{\infty}}=\lambda\left(\frac{\rho_{j} u_{j}}{\rho_{\infty} u_{\infty}}\right) \frac{r_{j}^{2}}{y_{5} z_{5}}
$$

For the twin-jet configuration, the concentration on the engine centerline is

$$
\frac{c_{E C L}-c_{\infty}}{c_{j}-c_{\infty}}=\lambda\left(\frac{\rho_{j} u_{j}}{\rho_{\infty} u_{\infty}}\right) \frac{r_{j}^{2}}{y_{5} z_{5}}\left\{1+\exp \left[-\lambda\left(\frac{E C L}{y_{5}}\right)^{2}\right]\right\}
$$

For an alrcraft with (m) engines, in regions where the horizontal half-value width is much larger than the engine separation distance, the centerline concentration difference ratio and the horizontal and vertical half-value widths are related by

$$
\frac{c_{E C L}-c_{\infty}}{c_{j}-c_{\infty}}=(m) \lambda\left(\frac{\rho_{j} u_{j}}{\rho_{\infty} u_{\infty}}\right) \frac{r_{j}^{2}}{y_{5} z_{5}}
$$

The independence of the plume size on the alrcraft initial conditions is an important result because to establish the dilution at any point in the far-wake region, only the plume size relations, Equations (13) and (14), and the plume sizeconcentration relation, Equation (17), are required. In the near-wake region, where the plume size is not independent of the initial conditions, a different plume size and/or concentration difference curve is required for every afrcraft and flight condition of interest.

\section{Results and Discussion}

The transition in the plume width from the near wake (jet dominant) to the far wake (atmospheric dominant) 18 shown in Figures 1 and 2 for the engines and filght conditions examined in Reference 2. In addition to the curves for the large (GE4) and small (J85) engines the solution for the plume width for dispersion from a point source moving with the aircraft is given. Vertical dispersion is shown on Figure 1 whereas horizontal dispersion 1s shown on Figure 2. The centerline concentration corresponding to the plume size may be obtained using Equation (15) and the initial conditions given in Reference 2 .

In Reference 2, concentrations of $\mathrm{CO}, \mathrm{NO}_{\mathrm{X}}$, and $\mathrm{O}_{3}$ measured in the wake of an F104 aircraft at distances from 2 to 10 kilometers behind the F104 are presented and compared with concentrations calculated using the near wake disperston model. Concentrations for this case calculated using the present method (with atmospheric dispersion effects Included) are identical to those shown in Reference 2 over most of the range of the flight experiment. Only at the largest distances, $-10 \mathrm{~km}$, are the calculated concentrations different from those given in Reference 2. At that distance, the region of atmospheric effects has just begun, and the dispersion is slightly more rapid than predicted with the near-wake model.

Calculations have been performed for exhaust dispersion and dilution for a YF-12 aircraft flying at Mach $2.8,19.8 \mathrm{~km}$ altitude, with and without afterburning and for a hypothetical four engine supersonic transport alrcraft of the Boeing SST configuration flying at Mach $2.7,19.8 \mathrm{~km}$ altitude with maximum afterburning.

The horizontal and vertical plume half-value widths for these are shown in Figure 3 for the YF-12 and Figure 4 for the SST. Also shown is the horlzontal half-width plus the outboard-engine-toalrcraft-centerline distance since this is a measure of the overall width of the wake. Atmospheric dispersion effects first appear at approximately 1 minute after alrcraft passage. For times on the order of an hour or greater, the corresponding widths for the YF-12 and the SST are identical. As reported by Walton (3) the horizontal diffusivity approaches its asymptotic value in from 5 to 10 days; as this occurs the horizontal width growth becomes proportional to $(t)^{1 / 2}$ indicative of the constant diffusivity. Since the asymptotic value of the vertical diffusivity is several orders of magnitude smaller than the horizontal value, the vertical diffuston is never really scale-dependent and the jet solution, width $\sim(t)^{1 / 3}$, blends smoothly into the constant diffusivity solution, width $\sim(t)^{1 / 2}$

The concentration difference ratios as a function of time since aircraft passage for the YF-12 and SST are shown in Figure 5. For times less than 1 minute the dispersion is dominated by jet effects and the centerline concentration difference ratio decrease is rapid initially, blends into the asymptotic $(t)-2 / 3$ result, and tends toward a $t-1 / 2$ decay as the exhaust jets merge. More rapid dilution occurs when the atmospheric dispersion is domInant as the eddy diffusivity is increasing with time. For times from a few minutes to a few days after alrcraft passage, the concentration difference ratio is proportional to $t^{-2}$. When the hor1zontal diffusivity reaches its asymptotic value, the concentration difference ratio is proportional to $1 / t$.

It has been suggested that the turbulent diffusion in the stratosphere is not as great as represented by the dissipation raje and asymptotic diffusivities used by Walton. (3) In Particular Lilly (c1ted in Ref. 4). suggests $D=2 \times 10^{-5} \mathrm{~m}^{2} / \mathrm{sec}^{3}$ and Hilst (5) suggests $K_{y}=10 \mathrm{~m}^{2} / \mathrm{sec}$ and 
$K_{z}=0.1 \mathrm{~m}^{2} / \mathrm{sec}$. Th1s value of $K_{y}$ removes the contributions of "meandering," i.e., turbulence of a scale greater than the width of the plume. It is not within the scope of the present paper to suggest values appropriate for stratospheric dispersion, however, it is interesting to note the significant differences in the predicted dispersion obtained by using the above parameters instead of those used in Reference 3.

In Figure 6 the calculated plume half-value widths for the YF-12 at Mach $2.8,19.8 \mathrm{~km}$ altitude, with maximum afterburning are shown for both sets of stratospheric dispersion parameters. The smaller atmospheric dissipation rate and asymptotic diffusivities result in prediction of smaller plume dimensions in the region where atmospheric effects predominate. Also, the effect of the smaller dissipation rate is to shift the onset of atmospheric effects to larger times after fly-by.

The centerline concentration difference ratios, for the YF-12 flight condition, calculated using both sets of stratospheric dispersion parameters are shown in Figure 7. Significantly less dilution is predicted using the smaller dispersion parameters; for example, at one day after aircraft passage the centerline concentration difference ratio predicted using $D=2 \times 10^{-5} \mathrm{~m}^{2} / \mathrm{sec}^{3}, \mathrm{~K}_{\mathrm{y}}=10 \mathrm{~m}^{2} / \mathrm{sec}$, and $\mathrm{K}_{\mathrm{z}}=0.1 \mathrm{~m}^{2} / \mathrm{sec}$ is 240 times the value predicted using $D=2 \times 10^{-4} \mathrm{~m}^{2} / \mathrm{sec}^{3}, \mathrm{~K}_{\mathrm{y}}=5 \times 10^{5} \mathrm{~m}^{2} / \mathrm{sec}$, and $K_{z}=1 \mathrm{~m}^{2} / \mathrm{sec}$.

The concentration of any desired species on the plume centerline can be obtalned from the centerline concentration difference ratio and the known (or estimated) ambient concentration and the concentration of the species in the engine exhaust. off-centerline concentrations can be obtained also by using the half-value width relations in addition to the above since the distributions are assumed to be Gaussian.

\section{Summary of Results}

A method is presented for calculating the dispersion of any conserved species in jet aircraft exhaust plumes at cruise conditions. The method is appropriate for estimating the dilution of the exhaust from aircraft passage through times on the order of weeks thereafter.

In the near wake of the aircraft, the mixing is dependent on the aircraft infitial conditions, and the flow from each engine is modeled as a round jet in a parallel flow. The velocity and concentration profiles are assumed to be Gaussian, and the eddy viscosity is assumed to be directly proportional to the engine alrflow plus the absolute value of the entrainment mass flow and inversely proportional to the jet width. The concentration decay is rapid inftially, but approaches the solution for a small excess jet as distance from the aircraft increases.

Multiple engine alrcraft are treated by superimposing the solutions from the several engines.

At some downstream distance, the eddy viscosity calculated For the jet flow becomes less than the eddy viscosity due to scale-dependent diffusion in the atmosphere. When this occurs, atmospheric effects are assumed to be dominant and the scaledependent eddy viscosity is used. The solution in

this region approaches that for dispersion from a point source moving with the aircraft. Since horizontal dispersion is known to be more rapid than vertical dispersion, a bivariant Gaussian distribution for species in the plume is assumed.

In the far wake region of the flow, the plume size is Independent of aircraft configuration and engine operating conditions. The dilution at any location can be determined from the plume size, engine alrflow, number of engines, flight speed, and altitude. Calculations for exhaust dispersion and dilution are presented for a YF-12 and a hypothetical SST aircraft at high altitude supersonic flight conditions.

\section{Appendix - Symbols}

$c_{c}$ centerline concentration - single engine

$c_{E C L}$ concentration on engine centerline for multiple engine alrcraft

$c_{j}$ jet exhaust concentration

$c_{\infty}$ ambient concentration

D atmospheric dissipation rate $[=] \mathrm{m}^{2} / \mathrm{sec}^{3}$

ECL distance between engines for multiple engine aircraft

dispersion coefficient $[=] \mathrm{m}^{2} / \mathrm{sec}$

stratospheric diffusivity, $=\mathrm{K}$ for vertical diffusion, $=\mathrm{K}_{\mathrm{y}}$ for horizontal diffusion

number of engines

radius

jet exhaust radius

half-value radlus where $\left(c-c_{\infty}\right) /\left(c_{c}-c_{\infty}\right)=$

0.5

plume centerline veloclty

jet exhaust velocity

streamwise velocity

flight speed

time after aircraft passage, $t=x / u_{\infty}$

streamwise distance

horlzontal distance; $(y=0)=$ aircraft center-

ine for multiple engines, = engine center-

ine for single engines

$\mathrm{y}_{5}$

2

$\mathbf{z}_{5}$

$\varepsilon$

$\lambda$

p

o horizontal half-value width

vertical distance

vertical half-value width

eddy viscosity [=] kg/(msec)

In 2

gas density

plume width standard deviation $=r_{5} / \sqrt{2 \lambda}$ 


\section{REFERENCES}

1. Hoshizak1, H., Cont1, R. J., Anderson, L. B. Redler, K. 0., and Meyer, J. W., "Study of High-Alt1tude A1rcraft Wake Dynamlcs," DOT-TST90-3, AD-754918, Dec. 1972, Lockheed M1ssiles and Space Co., Palo Alto, Calif.

2. Holdeman, J. D., "D1spersion of Turbojet Engine Exhaust in F11ght," TN D-7382, 1973, NASA, Cleveland, OhIo.

3. Walton, J. J., "Dispersion of Exhaust Plumes in the Stratosphere," Paper 73-532, June 1973, AIAA, New York, N.Y.

4. H1lst, G. R., and Donaldson, C. duP., "PrelimInary Estimates of the Fate of SST Exhaust Materials using a Coupled Diffuston/Chemistry Model," Paper 73-535, June 1973, AIAA, New York, N.Y.

5. H1lst, G. R., Donaldson, C. duP., and Cantiliano, R., "Some Analyses of the Chemistry and Diffusion of SST Exhaust Materlals During Phase III of the Wake Perlod," July 1973, Aeronaut1cal Research Associates of Princeton, Inc., Princeton, N.J.; also CR-132323, 1973, NASA. 


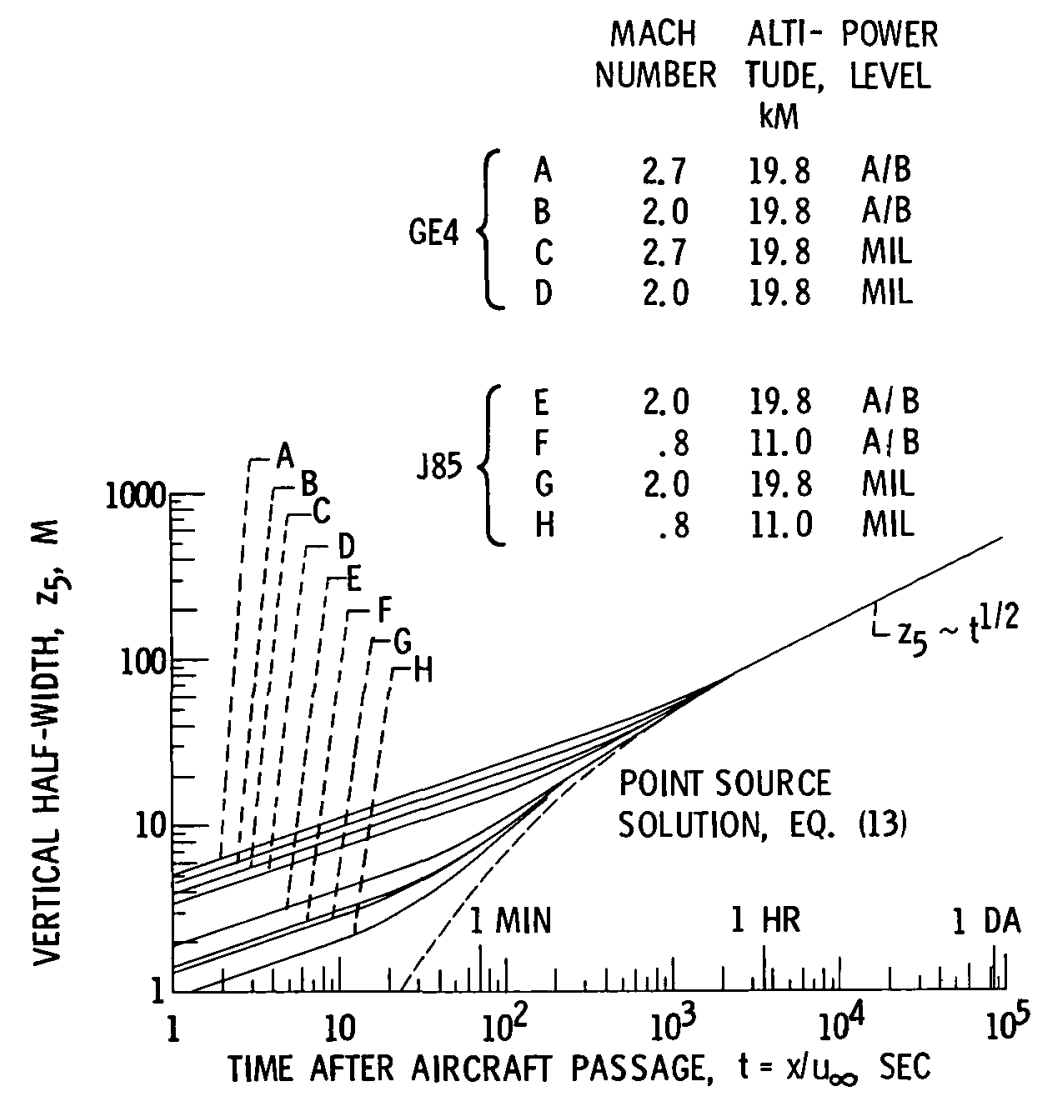

Figure 1. - Exhaust plume vertical half-width for GE4 and $\mathrm{J} 85$ engines at several flight conditions:

$D=2 \times 10^{-4} \mathrm{M}^{2} / \mathrm{sec}^{3} ; \mathrm{K}_{\mathrm{Z}}=1 \mathrm{M}^{2} / \mathrm{sec} ; \mathrm{A} / \mathrm{B}=$ maximum afterburning; $M I L=$ maximum power without afterburning.

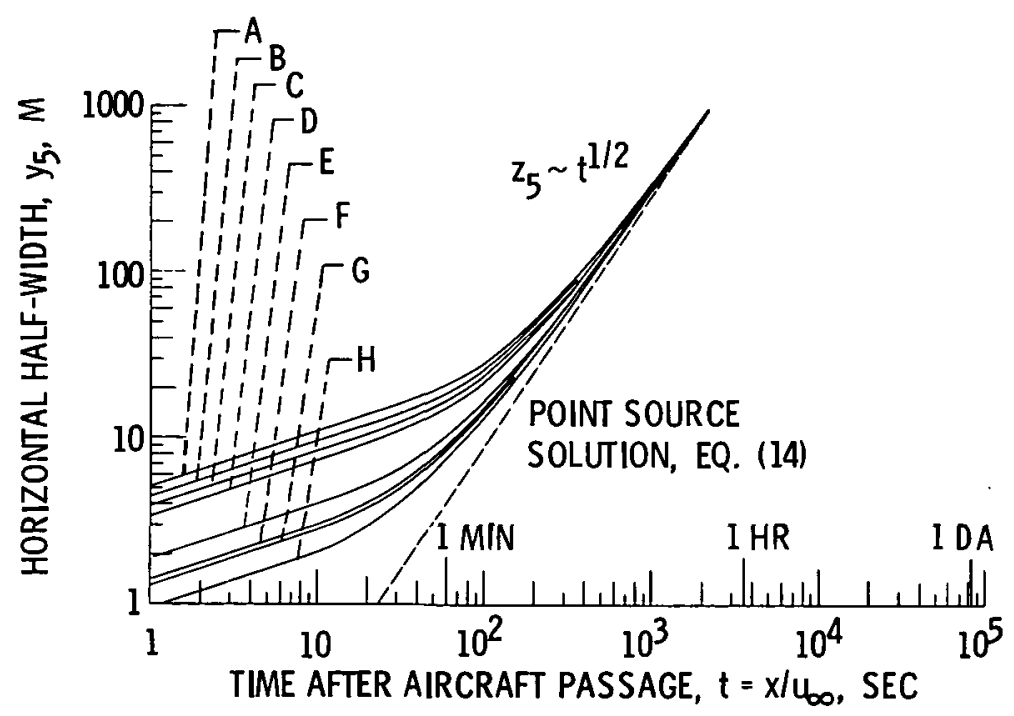

Figure 2. - Exhaust plume horizontal half-width for GE4 and J85 engines at several flight conditions: $D=2 \times 10^{-4} \mathrm{M}^{2} / \mathrm{sec}^{3}$; $\mathrm{K}_{\mathrm{y}}=5 \times 10^{5} \mathrm{M}^{2} / \mathrm{sec} ; \mathrm{A} B \mathrm{~B}=$ maximum afterburning; $\mathrm{MIL}=\operatorname{maxi}-$ mum power without afterburning. 


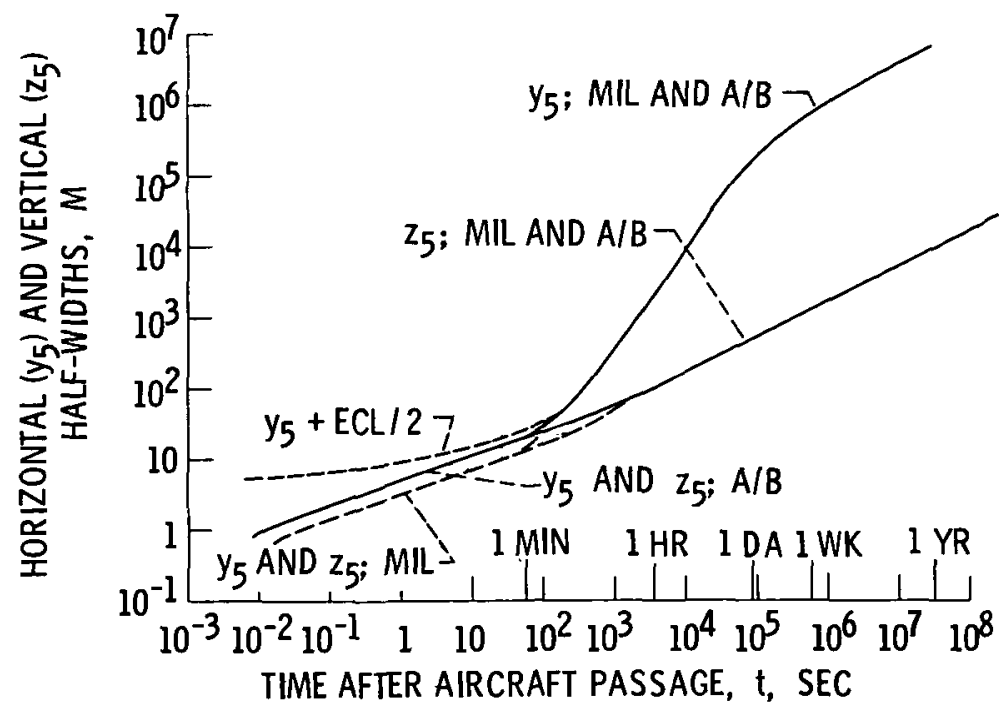

Figure 3. - Horizontal and vertical plume widt hs for $Y F-12$ aircraft $19.8 \mathrm{kM}$ altitude, Mach 2.8: $D=2 \times 10^{-4} \mathrm{M}^{2} / \mathrm{sec}^{3}$; $\mathrm{K}_{\mathrm{y}}=5 \times 10^{5} \mathrm{M}^{2} / \mathrm{sec} ; \mathrm{K}_{\mathrm{z}}=1 \mathrm{M}^{2} / \mathrm{sec}$.

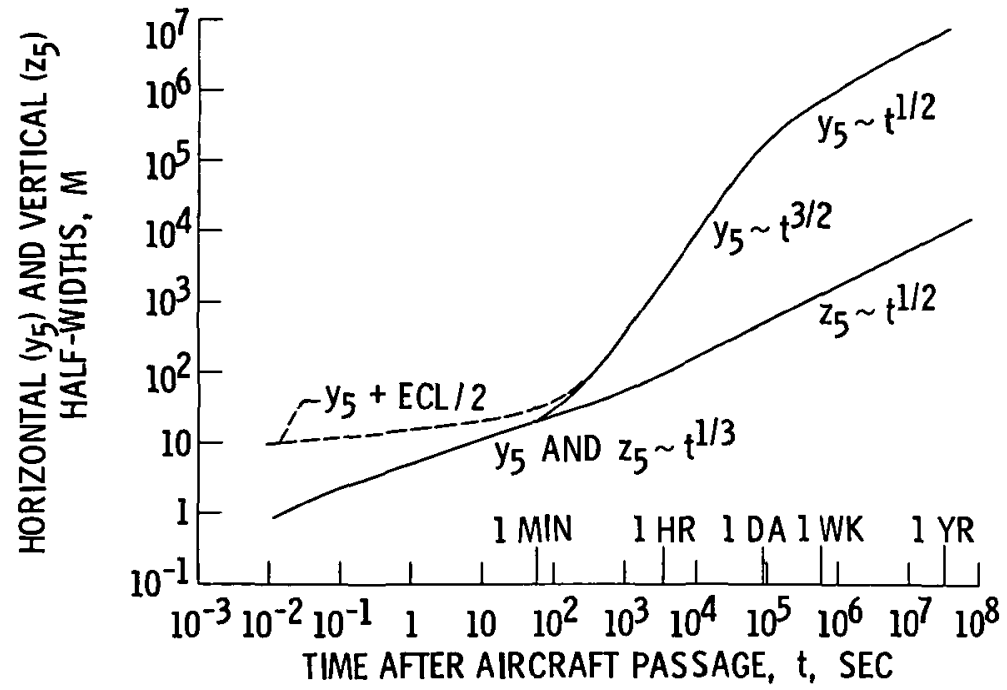

Figure 4. - Horizontal and vertical plume widths for SST, $19.8 \mathrm{kM}$ altitude, Mach 2.7, maximum afterburning: $D=2 \times 10^{-4} \mathrm{M}^{2} / \mathrm{sec}^{3} ; K_{y}=5 \times 10^{5} \mathrm{M}^{2} / \mathrm{sec}_{\text {; }}$ $K_{z}=1 M^{2} / \mathrm{sec}$. 


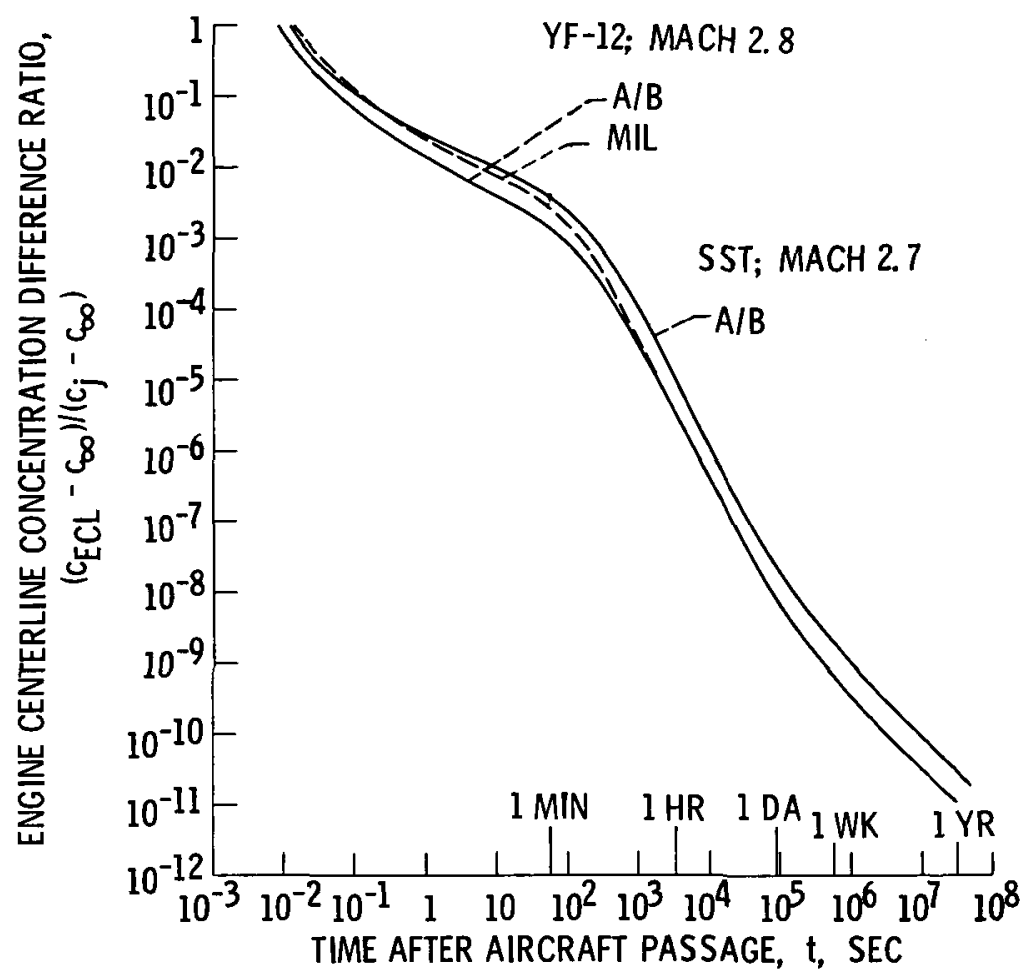

Figure 5. - Centerline concentration difference ratio for YF-12 and SST, $19.8 \mathrm{kM}$ altitude: $D=2 \times 10^{-4} \mathrm{M}^{2} / \mathrm{sec}^{3}$; $K_{y}=5 \times 10^{5} \mathrm{M}^{2} / \mathrm{sec} ; K_{z}=1 \mathrm{M}^{2} / \mathrm{sec}$. 

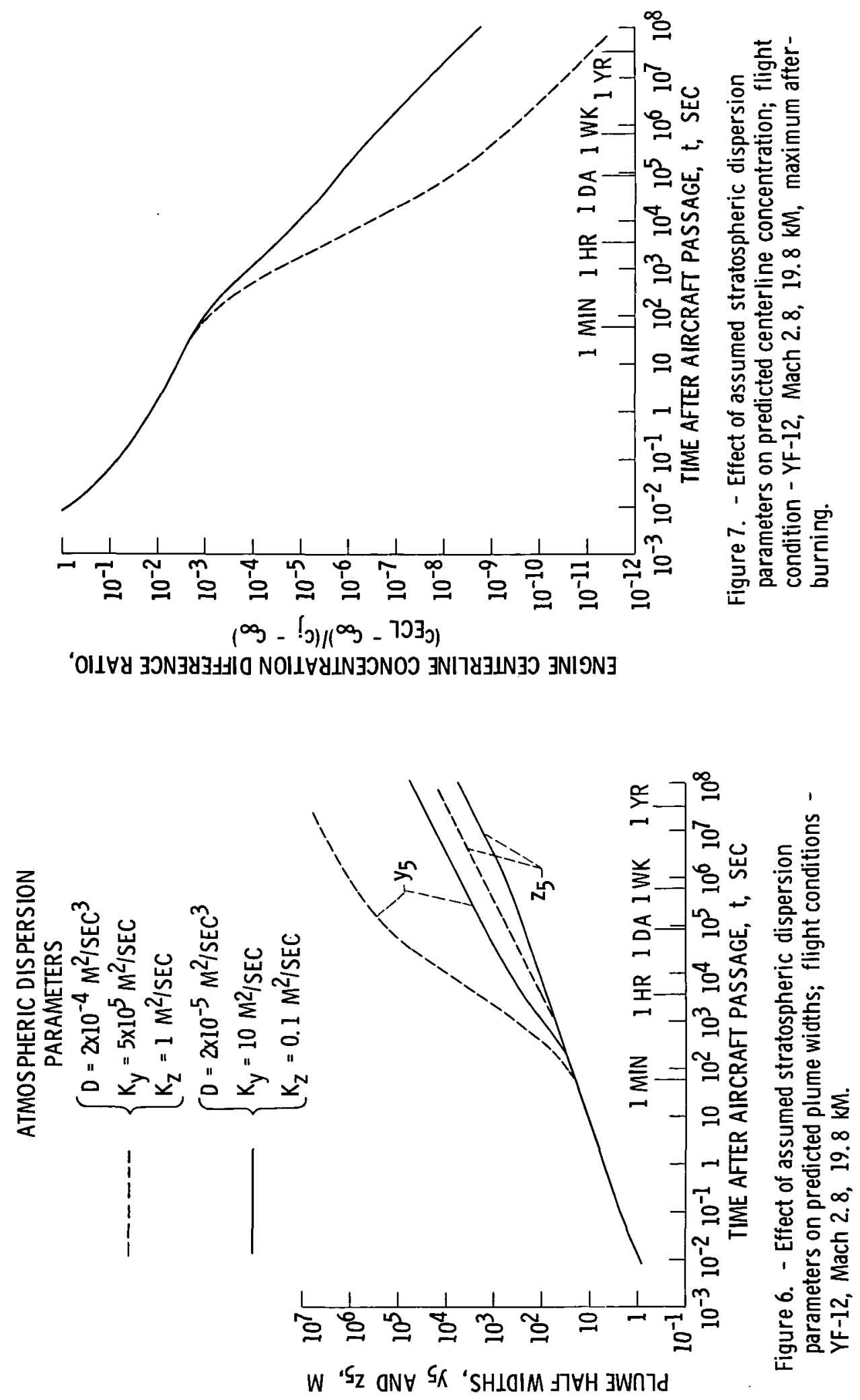